\title{
Psychosis incidence through the prism of early intervention services
}

\author{
J. B. Kirkbride, C. Stubbins, P. B. Jones
}

\section{Summary}

We know little about first-episode psychosis epidemiology beyond cities or when measured through early intervention in psychosis services. We present results from 18 months of the 3-year Social Epidemiology of Psychoses in East Anglia (SEPEA) study of incepted incidence observed through five early intervention services. We identified 378 eligible individuals (incidence: 45.1/100 000 person-years, 95\% Cl 40.8-49.9). Rates varied across these services, but were 2-3 times higher than those on which services were commissioned. Risk decreased with age, was nearly doubled among men and differed by ethnic group; doubled in people of mixed ethnicity but lower for those of Asian origin, compared with White British people. Psychosis risk among ethnic minorities was lower than reported in urban settings, which has potential implications for aetiology. Our data suggest considerable psychosis morbidity in diverse, rural communities

\section{Declaration of interest}

None.
Research into social factors in the aetiology of psychotic disorder has demonstrated notable variation observed by age and gender, ${ }^{1}$ cannabis use, ${ }^{2}$ immigrant status and ethnicity, ${ }^{3}$ and urban birth and upbringing. ${ }^{4}$ This epidemiological landscape is taken from studies predominately conducted in large cities. ${ }^{4}$ Less is understood about these risk markers outside of conurbations, where almost one-fifth of the English population lives. ${ }^{5}$ Delineating such epidemiology is also relevant to health services planning, particularly given recent reports that early intervention in psychosis services in both urban and rural English communities have observed psychosis rates up to three times higher than those upon which such services were first commissioned (i.e. 15/100 000 person-years). ${ }^{6,7}$ We present initial findings from the Social Epidemiology of Psychoses in East Anglia (SEPEA; www.sepea.org) study, a large, 3-year population-based first-episode psychoses study.

\section{Method}

The study methodology was based on the principles of a major epidemiological study of first-episode psychosis previously conducted in England, ${ }^{1}$ modified for use in early intervention services. We established a surveillance system to record sociodemographic and clinical data on all people aged 16-35 years resident within East Anglia, referred and accepted to our early intervention services with first-episode psychosis over 3 years, from 1 August 2009. ICD-10 clinical and research (OPCRIT) diagnoses for psychotic disorder (F10-39) are established at 6 months and 3 years after referral. Here, we report sample characteristics and initial incidence rates from the first 18 months of case ascertainment, using 2009 mid-term census population estimates as the denominator, adjusted for study duration. Poisson regression (Stata, version 11) explored covariate effects. Full method is given in the online supplement.

\section{Results}

Over the first 18 months of the study 510 people were referred to early intervention services in East Anglia with suspected firstepisode psychosis. In total $70 \%(n=357)$ met inclusion criteria during over 835000 person-years of follow-up. The main reason for exclusion was not meeting clinical criteria for psychosis at referral $(n=106 ; 20.8 \%)$ (see online Fig. DS1 for a complete breakdown of exclusions).

The crude incidence of clinically relevant psychotic disorder in East Anglia was estimated as 42.6/100 000 person-years (95\% CI 38.4-47.2). Rates were generally similar across services (online Table DS1), but were notably raised in Great Yarmouth and Waveney (54.9/100000 person-years; 95\% CI 39.9-75.4). Completed demographic data were available on 357 individuals $(92.4 \%)$ at the time of analysis. Median age at first presentation was similar for women (21.9 years, IQR $=18.2-25.8)$ and men (22.3 years, IQR $=19.3-26.7)$. Risk was elevated among men $(\mathrm{RR}=1.7,95 \% \mathrm{CI} 1.4-2.2)$, after adjustment for age and ethnicity. Our data also suggested risk differed by ethnicity. Compared with the White British group, people of Black $(\mathrm{RR}=1.8 ; 95 \% \mathrm{CI}$ 1.0-3.3) and mixed ethnicities ( $\mathrm{RR}=2.1 ; 95 \%$ CI 1.3-3.6) were at elevated risk of psychotic disorder after adjustment for age and gender. By contrast, people of Asian origin (including the Indian subcontinent and Southeast Asia) were at lower risk of psychosis compared with the White British group (adjusted $\mathrm{RR}=0.5,95 \%$ CI 0.3-0.9) (Table 1).

A total of $50 \%$ of our sample were unemployed at initial referral; $25 \%$ were in paid employment, $19 \%$ were students and $4 \%$ were unpaid family carers; information was missing from $2 \%$ of our sample.

\section{Discussion}

We identified variation in the incidence of psychosis in a diverse, mainly rural English region. The overall incidence was higher than typically reported in first-episode psychosis studies of the entire adult age range (16-64 years), but this is to be expected given our lower age limit (35 years) and the decline in risk with age. ${ }^{1}$ For comparison, the incidence for people 16-35 years old in the ÆSOP study varied from 32.0/100 000 person-years in Bristol to 74.0/100 000 person-years in south-east London, placing our estimates within this range. Nevertheless, observations from both SEPEA and ÆSOP are consistent with recent empirical data ${ }^{7}$ that the incidence of psychotic disorders seen through early intervention services is generally three times greater than the figure upon which such services were commissioned. ${ }^{6}$ This has 
Table 1 Sample characteristics and adjusted rate ratios in the SEPEA study at 18 months $^{\text {a }}$

Adjusted $^{\mathrm{C}}$

\begin{tabular}{lccc} 
Variable & $\begin{array}{c}\text { Participants, } \\
n(\%)\end{array}$ & $\begin{array}{c}\text { Denominator, } \\
n(\%)^{\mathrm{b}}\end{array}$ & $\begin{array}{c}\text { Adjusted }^{\mathrm{C}} \\
\text { relative risk } \\
(95 \% \mathrm{Cl})\end{array}$ \\
\hline Total & $357(100)$ & $838574(100)$ & -
\end{tabular}

Early intervention service

$(n=357)$

Cambridgeshire,

Peterborough \& Royston 122 (34.2)

Petert Norfor

Central Norfolk

Great Yarmouth

\& Waveney

$17(4.8)$

$306283(36.5)$

$91(25.5)$

$41765(5.0)$

$219860(26.2)$

Suffolk

$38(10.6) \quad 69218(8.3)$

Gender $(n=330)$

Women $\quad 115(34.8) \quad 405221(48.3) \quad 1$

Men

$215(66.2) \quad 433353(51.7) \quad 1.7(1.4-2.2)$

Age group $(n=330)$

16-17

$52(15.8) \quad 71929(8.6) \quad 1$

$18-19$

$53(16.1)$

20-24

$118(35.8)$

$88976(10.6) \quad 0.8(0.6-1.2)$

25-29

30-35

$\begin{array}{lll}73(22.1) & 213385(25.4) & 0.5(0.3-0.7) \\ 34(10.3) & 245127(29.2) & 0.2(0.1-0.3)\end{array}$

Ethnicity $(n=330)$

White British

White non-British

$261(79.1) \quad 671588(80.1) \quad 1$

Mixed ethnicity

Black

$21(6.4)$

$50882(6.1)$

$1.2(0.8-1.9)$

$15(4.5) \quad 17364(2.1) \quad 2.1(1.3-3.6)$

Asian

$12(3.6)$

$1.8(1.0-3.3)$

$12(3.6) \quad 69014(8.2) \quad 0.5(0.3-0.9)$

Other ethnicities

$9(2.7)$

$11255(1.3) \quad 2.3(1.2-4.5)$

a. Because the study is ongoing, detailed sociodemographic data were only available for a subset $(n=309)$ of the total incidence sample $(n=378)$. Thus, incidence rates were reported where we had data on the full sample $(n=378)$, with relative risks

reported from Poisson regression on demographic data for the subsample $(n=309)$.

b. Adjusted for duration of case ascertainment in each early intervention service

(18 months).

c. Adjusted for other variables in model (age group, gender and ethnicity).

important implications for mental health service provision. In our sample, age at first presentation was similar for both genders before 36 years old, a point easily overlooked in entire adult-onset samples, where median age at onset typically occurs a few years later for women ${ }^{1}$ as a result of a secondary peak of psychosis close to the time of menopause, ${ }^{8}$ not captured by our early intervention services data.

We also reported elevated psychosis risk for some minority groups in East Anglia, although not, even at the upper limit of the confidence interval, to the extent observed in more urban settings. ${ }^{3}$ Strikingly, relative risk estimates in people from Asian populations were half those observed in the White British group. Although we cannot exclude the possibility that these differences were explained by differential service utilisation, our findings are consistent with the possibility that migrant and minority groups in more rural communities may not be exposed to the same degree to the factors that drive elevated psychosis rates in cities. This hypothesis will be pursued in the full dataset, but there is already supporting evidence: cumulative social disadvantage and separation and loss events in childhood are reported to be associated with increased odds of psychosis for both White British and ethnic minority groups but the impact of such events appears to be more pervasive among some minority groups. ${ }^{9,10}$ If socioenvironmental exposures were also amplified in urban compared with rural populations, or led to greater stress responses in urban dwellers, as has been recently observed in a small sample of healthy adults, ${ }^{11}$ this could potentially explain the attenuation in elevated rates among rural minority populations.
Our initial data suggested that incidence rates were elevated in one of our services, and we will consider multivariate, multilevel explanations for this in our final data-set, including the possibility that the variation may be partially driven by service-side factors, such as the degree of active outreach provided by different services and the length of time services have been established. We will also be able to inspect differences in rates according to diagnostic subgroup and demographic factors not reported here (including country of birth, age at migration, generation status, occupation), compare clinical- and research-based diagnoses, and inspect the evolution of symptomatology in a first-episode sample over 3 years of treatment. Nevertheless, the incepted incidence rates assessed here through clinical early intervention services highlight a substantial burden of psychotic disorder beyond cities, and potentially provide important aetiological clues.

J. B. Kirkbride, PhD, C. Stubbins, BA(Hons), P. B. Jones, PhD, MRCPsych, Department of Psychiatry, University of Cambridge, Cambridge, UK

Correspondence: J. B. Kirkbride, Department of Psychiatry, University of Cambridge, Box 189, Addenbrooke's Hospital, Hills Road, Cambridge CB2 2QQ UK.Email: jbk25@cam.ac.uk

First received 29 Mar 2011, final revision 15 Jul 2011, accepted 28 Jul 2011

\section{Funding}

J.B.K. was supported by a Sir Henry Wellcome Research Fellowship from the Wellcome Trust (grant number WT085540), through which the SEPEA study was established. P.B.J. directs the NIHR Collaboration for Leadership in Applied Health Research and Care (CLAHRC) for Cambridgeshire \& Peterborough and is supported by NIHR grant RP-PG-0606-1335. The SEPEA study has been adopted by the Mental Health Research Network (MHRN).

\section{Acknowledgements}

The authors are grateful to the clinical services and staff participating in the SEPEA study, and the MHRN for their generous support.

\section{References}

1 Kirkbride JB, Fearon P, Morgan C, Dazzan P, Morgan K, Tarrant J, et al. Heterogeneity in incidence rates of schizophrenia and other psychotic syndromes: findings from the 3-center $\mathbb{E}$ ESOP Study. Arch Gen Psychiatry 2006; 63: 250-8.

2 Moore THM, Zammit S, Lingford-Hughes A, Barnes TRE, Jones PB, Burke M, et al. Cannabis use and risk of psychotic or affective mental health outcomes: a systematic review. Lancet 2007; 370: 319-28.

3 Cantor-Graae E, Selten J-P. Schizophrenia and migration: a meta-analysis and review. Am J Psychiatry 2005; 162: 12-24.

4 March D, Hatch SL, Morgan C, Kirkbride JB, Bresnahan M, Fearon P, et al. Psychosis and place. Epidemiol Rev 2008; 30: 84-100.

5 Department for Environment, Food and Rural Affairs. Statistical Digest of Rural England 2011. DEFRA, 2011.

6 Department of Health. Mental Health Policy Implementation Guide. National Health Service, 2001.

7 Cheng F, Kirkbride JB, Lennox BR, Perez J, Masson K, Lawrence K, et al Administrative incidence of psychosis assessed in an early intervention service in England: first epidemiological evidence from a diverse, rural and urban setting. Psychol Med 2011; 41: 949-58.

8 Grigoriadis S, Seeman MV. The role of estrogen in schizophrenia: implications for schizophrenia practice guidelines for women. Can J Psychiatry 2002; 47: 437-42.

9 Morgan C, Kirkbride J, Hutchinson G, Craig T, Morgan K, Dazzan P, et al Cumulative social disadvantage, ethnicity and first-episode psychosis: a case-control study. Psychol Med 2008; 38: 1701-15.

10 Morgan C, Kirkbride JB, Leff J, Hutchinson G, McKenzie K, Morgan K, et al. Parental separation, loss and psychosis in different ethnic groups: a case-control study. Psychol Med 2007; 37: 495-503.

11 Lederbogen F, Kirsch P, Haddad L, Streit F, Tost H, Schuch P, et al. City living and urban upbringing affect neural social stress processing in humans. Nature 2011; 474: 498-501. 